nent opening in the drum-head, exposing the stapes to view, division of the stapedius tendon, leaving the stapes to move freely, as a plunger, in its niche and the application, to the head of the stapes, with a supporting bearing upon the edge of the perforation in the drum-head, or upon the promontory, of a disk of stiff, sized paper, presenting to the sound waves a receptive surface of from four to ten or more times the superficial area of the base plate of the stapes, has effected a gradual mobilization evidenced by a progressive improvement in the hearing; in some of the cases thus studied it was necessary, in the earlier stages of the experiment, to resort to the direct application of the sound waves, but, in the majority of the selected cases, the sound waves of ordinary causation sufficed.

With a due appreciation of the proper apportionment of the motive force to be applied, and with a proper estimation of the injurious effects upon the perceptive organ of continuous loud sounds, coupled with a study of the accumulating records of various observers, it will be possible to release this subject from the bonds of clinical empiricism and place it upon its true basis as a therapeutic measure.

This much may be at least taken for granted, that the application of the major force exertable by the majority of aural massage instruments is objectionable and that such instruments should be used only under careful observation of their immediate effects, that phonomassage, of mechanical production, where abounding in metallic overtones, is objectionable because of its fatigue effect, and that the use of sound waves judiciously applied, with a sufficient knowledge of the existing aural condition, is a valuable supplement to the mechano-therapeutics of the ear.

In conclusion it may be said that application of sound waves to the ear for therapeutic purposes is the application of a form of massage commensurably proportionate to the delicacy of the sound transmitting structures of the middle ear and labyrinth.

That the prolonged use of tones of excessive amplitude and very low or very high pitch, whatever their beneficial mobilizing effect upon the sound transmitting apparatus of the middle ear may be, are open to the objection of their prejudicial effect upon the organ of perception.

That mechanical reproductions of speech are open to the objection of their accentuation of overtones and their presentation of a distortion of a familiar sound picture, that of the normal voice.

That the natural voice concentrated upon the ear is, both in its range and its volume, as well as in the more general exercise afforded by its complexly compounded sound waves and the readiness with which it may be uniformly applied, the safest and, in the end, the best form of sound source for phonomassage of the ear.

Cholera.- It is reported that cholera has appeared at Manila, but no anxiety is entertained.

\section{A METHOD FOR THE DIFFERENTIAL STAINING OF BLOOD PLATES.}

BY JAMES HOMER WRIGHT, MD., S.D.,

Director of the Pathological Laboratory of the Massachusetts General Hospital; Instructor in Pathology, Harvard Medical School.

IN a recent paper upon "The Origin and Nature of the Blood Plates," published in this JouRnal, ${ }^{1}$ I described the results obtained by means of a special staining method for these corpuscles, but I did not describe the method in detail.

I have recently improved it in some particulars and it may be now described as follows:

Fixation of perfectly fresh tissue in methyl alcohol; dehydration by acetone; clearing by xylol; imbedding in paraffin. Sections of $2 \mu$ thickness, for thicker sections do not give the best staining reactions.

Staining. - The sections are stained while affixed to a coverglass by Wright's blood staining fluid $^{2}$ in the same manner as a blood smear.

1. A sufficient quantity of this staining fluid is placed on the coverglass to cover it and then an equal quantity of water is added to the staining fluid drop by drop. The mixture remains on the preparation ten minutes.

2. Rinse in water.

3. Immerse in a 1 to 5000 aqueous solution of sodium hydrate for five seconds.

4. Wash in water.

5. Dehydrate with acetone.

6. Clear with oil of turpentine.

7. Mount in turpentine colophonium.

A most important feature of the method is the use of acetone and oil of turpentine in dehydrating, clearing and mounting, for these do not destroy the characteristic staining as do other similar reagents that I have experimented with.

The spleen of a young kitten is the most favorable object for the demonstration of the characteristic staining and structure of the blood plates and their origin from the giant cells as described in my paper referred to above, because this organ in this kind of animal contains many giant cells and also very many blood plates which are especially large in the cat.

Because the blood plates by this method exhibit peculiarities of staining and structure which permit them to be definitely recognized as blood plates and to be clearly distinguished from all other histological elements, I have been able to show that the so-called granular material seen in sections of mixed thrombi in man consists exclusively of blood plates and their fragments without any admixture whatever of fragments of red blood corpuscles.

\section{THE OCCURRENCE OF OCCULT HEMORRHAGES IN TYPHOID FEVER.* \\ BY WILDER TILESTON, M.D.}

IN view of the recent advances in the detection of small traces of blood in the feces, it has seemed worth while to examine the stools in typhoid

1 Boston Medical and Surgical Journal, June 7, 1906.

2 Journ. Med. Research, vii, 138, January, 1902

* From the Clinico-Pathological Laboratories of the Massachusetts General and Boston City Hospitals, Drs. J. H. Wright, F. B. Mallory,
directors. 
fever, in order to determine the frequency with which "occult hemorrhages" occur; $i$. e., those not evident to the naked eye. I have, accordingly, during the fall of 1905 and the following winter, examined the stools in 68 cases of typhoid by means of the guaiac and aloin tests for blood pigment.

The technique employed was essentially that of Boas.' About 5 cc. of liquid feces are taken, or, if the stool is solid, a piece the size of the end of the thumb is mixed with enough water to render it semi-fluid. The fat is first extracted with 20 cc. ether. To the residue is then added one third to one half its volume of glacial acetic acid, the two are thoroughly mixed and allowed to stand five minutes.

The next step is extraction with 8 to 10 cc. ether for fifteen minutes. The ethereal extract is divided into two equal portions, one for the guaiac, the other for the aloin test. The guaiac test is performed as follows: to the ethereal extract are added, first, a few grains of powdered resin of guaiac; next, 20 drops of old oil of turpentine; the mixture is then well shaken. A positive test is indicated by the appearance of a blue or violet color within fifteen minutes. Following the majority of writers on the subject, I have considered colors ranging from purple to pure blue as positive, all others as negative.

For the aloin test, a fresh solution was always used, prepared by dissolving a knife-point of aloin (Merck) in $\frac{1}{2}$ test tube $70 \%$ alcohol. Of this solution 2 or 3 cc. are added to the ethereal extract, and then 20 drops of old oil of turpentine. If positive, a color varying from rose to cherryred, should appear within fifteen minutes.

In the following series, only those cases are considered positive, which gave positive reactions with both guaiac and aloin. A careful examination to exclude other sources of hemorrhages (nose-bleed, hemorrhoids, etc.) was made in all positive cases, and all articles were excluded from the diet which might give rise to error.

Description of the Cases. - Sixty-eight consecutive cases were taken. The majority did not appear very sick, and, in fact, a comparison with the figures for the year 1905 at the two hospitals shows that the mortality was decidedly low. The death-rate for the whole year (536 cases) was $11 \%$, that for this series was only $7 \%$.

Results of Examinations. - Eighteen cases out of the 68 gave positive tests at some time during the course of the disease. One of these was complicated with enteritis, to which the presence of blood was probably due; there remain 17 , or $25 \%$, which showed the presence of occult blood. In all, 422 stools were examined, of which 42 were positive, $i$. e., $10 \%$.

A division into groups, according to the severity of the disease, gave the following results:

Fifteen mild cases, 3 positive, $20 \%$.

Thirty-nine moderately severe, 10 positive, $26 \%$.

Fourteen severe or fatal, 5 positive, $36 \%$.

Boas, I.: Ueber die Diagnose des Ulcus Ventriculi, etc. Deutsche med. Woch., 1903, p. 865.
One sees that the frequency of positive results increases with the severity of the disease.

A glance at the subjoined table shows that the occult hemorrhages, beginning in the second week, persist through the following week, and reach their maximum in the fourth week. The fifth week, usually afebrile, gives the lowest figures, $4.6 \%$. During the relapse, one case only was positive, or $9 \%$.

\begin{tabular}{|c|c|c|c|c|c|}
\hline Period. & $\begin{array}{c}\text { No. of } \\
\text { Cases. }\end{array}$ & $\begin{array}{c}\text { No. of } \\
\text { Positive } \\
\text { Cases. }\end{array}$ & $\begin{array}{l}\text { Per cent } \\
\text { of Positive } \\
\text { Cases. }\end{array}$ & $\begin{array}{c}\text { Total No. } \\
\text { of } \\
\text { Exams. }\end{array}$ & $\begin{array}{c}\text { No. of } \\
\text { Positive } \\
\text { Exams. }\end{array}$ \\
\hline 1st week, & 2 & 0 & 0 & 2 & 0 \\
\hline 2d week, & 29 & 5 & 17 & 67 & 7 \\
\hline 3d week, & 48 & 8 & 17 & 137 & 17 \\
\hline 4th week, & 40 & 9 & 22.5 & 99 & 13 \\
\hline 5th week, & 22 & 1 & 4.6 & 46 & 1 \\
\hline Relapse, & 11 & 1 & 9 & 37 & 2 \\
\hline
\end{tabular}

Five cases of typhoid in children under the age of fourteen years were examined, with positive result in one $(20 \%)$. This patient, a child of nine, showed traces of blood on two occasions, on the eleventh and twenty-fifth days of the disease, respectively.

A study of the positive cases brought out the following facts. The temperature, on the day of positive reactions, was considerably elevated in most cases; in 5, however, it had nearly, or quite, reached normal. Diarrhea was present in 11 , absent in 6 cases. The number of positive results was not large, averaging 2.4 per case. It is probable, however, that a daily examination of the stools would have given higher figures.

The relation to gross hemorrhages is interesting, but disappointing. Hemorrhages occurred in 7 cases $(10 \%)$; in 6 of these, although stools were examined in all within the preceding twenty-four hours, the tests were negative. In one case only were positive results obtained before the bleeding became manifest.

The prognosis was certainly not unfavorably affected by the occurrence of occult hemorrhages, for the mortality of the positive cases was $5.5 \%$, while that of the whole series was $7 \%$. Of the 6 cases in which gross hemorrhages occurred without premonitory positive tests, 4 died.

The Literature. - Joachim, ${ }^{2}$ in 1904, was the first to publish results of guaiac reactions on the stools of typhoid patients. He found positive tests in 2 out of 6 cases (33\%).

Tedeschi ${ }^{3}$ examined "many" cases, and never obtained positive results in the first two weeks. In the third week, 3 cases out of 24 were positive $(12.5 \%)$. One case, during a severe relapse, showed positive reactions on three successive days; on the fourth day, intestinal hemorrhage took place. Of 3 cases with peritonitis, 2 were positive, the third, said to be peritonitis "by propagation," was negative (no autopsy or operation).

In an interesting paper, Petracchi ${ }^{4}$ gives his results based on a material of 18 cases, with examinations every other day. Of these, 8 were positive (44\%). He includes as such, how-

2 Joachim, G.: Berliner klin. Woch., 1904, p. 466.

3 Tedeschi, E. Cronaca della Clinica Medica di Genova, 1904 p. 370.

Petrachi, C.: Zeitschr, f. klin. Med., 1905, No. 56, p. 167 
ever, some that were positive to aloin, but negative to guaiac. Of the 10 negative cases, all recovered, whereas 3 of the 8 positive cases died. In 4 cases a gross hemorrhage followed one to five days after the first appearance of a positive reaction. In 5 cases the appearance of the aloin reaction was accompanied by a rise in the pulse-rate of 10 to 22 beats; in 3 of these a large hemorrhage followed. He regards this combination as a very important means of foretelling hemorrhage.

Zuccola ${ }^{5}$ reports 23 cases of typhoid fever, with daily examinations of the stools. His results are unique, for he obtained positive tests in every case, and frequently throughout the course of the disease. He found in most cases early hemorrhages at the end of the first and beginning of the second week, corresponding to the stage of infiltration; later, "occult" hemorrhages were always present during the third week, at the time of the separation of the sloughs. They were more extensive and prolonged than the early ones. Positive tests were obtained as late as the thirty-eighth day.

The statements of Steele ${ }^{B}$ are in striking contrast with those of Zuccola; 3 cases positive out of 19 , or $16 \%$. The positive reactions were all in the second week, and occurred but once in each of the 3 cases; 92 stools were examined in all.

Cattaneo ${ }^{7}$ examined 3 cases, with positive results in one. In a fourth case, the reactions were positive for seventeen days after a large hemorrhage.

The conflicting statements with regard to the presence of blood in the stools in typhoid, as shown by the guaiac and aloin tests, are probably due to several causes. In the first place, they may be due to variations in the severity of the epidemic; for instance, Petracchi, with a mortality of $17 \%$, found $44 \%$ positive; I had $25 \%$ positive, with a death-rate of $7 \%$. Secondly, differences in technique and in the frequency with which the stools were examined are of importance; and thirdly, the personal equation, which is apt to be large when a color reaction is in question. The difficulty in determining the exact date of onset of the disease explains the discrepancies in the statements as to the period of greatest frequency of occult hemorrhages.

Conclusions. - From my own work and that of others, it would appear that:

(1) Occult hemorrhages may be detected by the guaiac and aloin tests in about $25 \%$ of all cases of typhoid fever.

(2) The application of these tests is of little value as a means of foretelling gross hemorrhages.

(3) It is of very little value in diagnosis, owing to the inconstancy and comparatively late appearance of positive reactions.

It seems probable that more complicated chemical methods would show a larger percentage of positive results, for the separation of the sloughs must always be attended by some

Zuccola, P. F.: Clinica Medica Italiana, April, 1905, p. 193.

- Steele, J. D.: Am. J. Med. Sc., July, 1905, p. 49.

7 Cattaneo, F.: Revista Critica di Clinica Medica, November, 1905 , p. 713 . hemorrhage, however slight. Such methods, however, will probably consume too much time to be applicable to clinical work, and would be of doubtful practical value, owing to the increased difficulty of excluding hemorrhage from other sources.

For kind permission to make use of the material, I wish to thank Drs. R. H. Fitz, J. J. Minot, F. C. Shattuck and H. F. Vickery, of the Massachusetts General Hospital, and Drs. G. B. Shattuck and J. L. Ames, of the Boston City Hospital.

\section{SORE THROAT. ETIOLOGY AND THERAPEUSIS.}

BY EDMUND D. gPEAR, M.D., BOSTON.

THE first tonsil is a distinct glandular organ situated in the naso-pharynx and was clearly described by Luschka, whose name it bears.

Whenever one discovers an acute inflammation of the throat he must predicate the presence of a chronic affection of the same. Dividing, by age limit, the usual types of sore throat into those of youth, of middle age, and of senility, it is fair to assume that the infections of childhood shall furnish the greatest proportion of cases.

It is proposed to call attention principally to those forms of disease of the throat of which the most important clinical symptom is dysphagia. In this connection it is made evident that the first subjective evidence of infection will be manifested to the patient by pain during the act of swallowing. This dysphagia, at first noticed only as a slight lameness of the throat muscles, rapidly increases to localized soreness, describable as a "spot," usually to the side of the pharynx, and coincident with the usual site of infection in the naso-pharynx.

In explanation of the actual cause of sore throat, it will be recalled that the muscular tissues in this region spread out over the surface of the bones forming the pharynx, and grow thinner as they approach the caput pharyngis, over the basilar process of the occipital bone. The swelling of these tissues, together with infiltration into their substance by the products of inflammation, causes pressure upon underlying nerve filaments, which become more strongly compressed by the fibers of the pharyngeal constrictors contracting during deglutition.

As stated above, and bearing upon the question of early abortive treatment of tonsillar abscesses, it is noted that "sore throat" may be quite noticeably severe many hours before local objective symptoms are discoverable by ordinary inspection of the fauces. As the disease advances the infiltration of the deeper muscular structures of the fasciæ and of lymphatic vessels increases so that movements of the head or neck produce distress and pain. In very many severe cases of infection, however, especially in those of incipient tuberculosis and of influenza, there is great disparity between the evident intense constitutional infection and the local disease at the site of 